\title{
Detection of asymptomatic retinitis pigmentosa
}

\author{
Chiranjeev Singh Jhajj ${ }^{1}$, K Kalaivani ${ }^{2}$, B Saranya ${ }^{3}$, P Deepthika ${ }^{1}$ \\ From ${ }^{1}$ Resident, ${ }^{2}$ Professor and Head of Department, ${ }^{3}$ Assistant Professor, Department of Ophthalmology, Vinayaka Missions Medical College and \\ Hospitals, Karaikal, Pondicherry, India
}

\begin{abstract}
Retinitis pigmentosa (RP) is a heterogeneous group of progressive hereditary disorders that affect photoreceptors and retinal pigment epithelial function. It is one of the most common hereditary fundus dystrophies. We report the case of a 30-year-old female with RP, who presented to our outpatient department with a complaint of defective vision of both eyes for the past 2 years. Further detailed history revealed the presence of nyctalopia since childhood with a positive family history of nyctalopia in younger brother in a family of two sons and one daughter. It was followed by a complete ophthalmological workup in which the anterior segment examination was found to be normal and fundus examination revealed waxy pallor, arteriolar attenuation, and bony spicules in both eyes. Visual fields showed tubular fields in both eyes. Hence, a diagnosis of typical RP of both eyes was made which initially looked like a case of simple refractive error only. The patient was advised of family screening and 6 monthly follow-ups.
\end{abstract}

Key words: Asymptomatic, Bony spicules, Retinitis pigmentosa

$\mathrm{R}$ etinitis pigmentosa (RP) is a heterogeneous group of progressive hereditary disorders that affect photoreceptors and retinal pigment epithelial function, which are associated with progressive cell loss and eventually atrophy of several retinal layers. It is one of the most common hereditary fundus dystrophies. The worldwide prevalence of RP is about one in 4000 while the prevalence of RP has been found to be 1:600-750 in South and Central India [1]. The disease can be inherited as an autosomal-dominant (about $30-40 \%$ of cases), autosomal-recessive (50-60\%), or X-linked (5-15\%) trait [2-4].

Usually, RP is confined to the eye, however, some $20-30 \%$ of patients are associated with non-ocular diseases like Usher's syndrome, which is associated with hearing impairment and is the most frequent form of syndromic RP [5]. The patients of RP typically lose night vision in adolescence, side vision in young adulthood, and central vision in later life because of the progressive loss of rod and cone photoreceptor cells. Thus, there is a need for early detection of the disease to improve the quality of life of patients and prevent complications of visual handicap or blindness among patients with a family history of RP. Similarly, genetic counseling is advised for future pregnancies to decrease the chances of developing RP.

\section{Access this article online}

Received - 30 April 2021

Initial Review - 15 May 2021

Accepted - 25 May 2021

DOI: $10.32677 /$ IJCR.2021.v07.i06.003

\section{CASE REPORT}

A 30-year-old female presented to our outpatient department with a chief complaint of defective vision of both eyes for the past 2 years. The defective vision was painless with a gradual onset and progressive in nature. There was the presence of nyctalopia since childhood with no history of usage of spectacles. There was no history of diabetes, hypertension, or history of consanguinity. There was a positive family history of nyctalopia in the younger brother in a family of two sons and one daughter. The patient has one son, with no complaint of nyctalopia and no evidence of RP. Similarly, the patient's grandparents had no complaint of nyctalopia. This pattern on the pedigree chart gave us an autosomal recessive pattern (Fig. 1).

General examination was normal and vitals were within normal limits. Eyes were orthophoric with no face turn or head tilt. The best-corrected visual acuity was $6 / 9 \mathrm{p}$ with $-1.75 \mathrm{Dcyl} \times 10^{\circ}$ in the right eye and $6 / 6$ with $-1.25 \mathrm{Dcyl} \times 160^{\circ}$ in the left eye. Anterior segment examination was normal. Slit-lamp biomicroscopy with 90 D showed waxy pallor, obliterated cup, arteriolar attenuation, and bony spicules in the periphery in both eyes (Fig. 2). Intraocular pressure was normal in both eyes. Visual fields on perimetry were done and the findings were suggestive of tubular vision in both eyes due to damage to rod photoreceptors in the mid-periphery (Fig. 3). Based on the clinical findings, a diagnosis of typical RP was made though initially was thought to have only refractive error only.

Correspondence to: Dr. Chiranjeev Singh Jhajj, Department of Ophthalmology, Vinayaka Missions Medical College and Hospitals, Karaikal, Pondicherry, India. E-mail: chiranjeevjhajj@gmail.com

(c) 2021 Creative Commons Attribution-NonCommercial 4.0 International License (CC BY-NC-ND 4.0). 
The patient refractive error was corrected and a strict 6 monthly follow-up was advised. Screening of the patient's sibling and son was done. Only her younger brother was diagnosed with RP.

\section{DISCUSSION}

RP patients typically lose night vision in adolescence, side vision in young adulthood, and central vision in later life because of progressive loss of rod and cone photoreceptor cells. In our

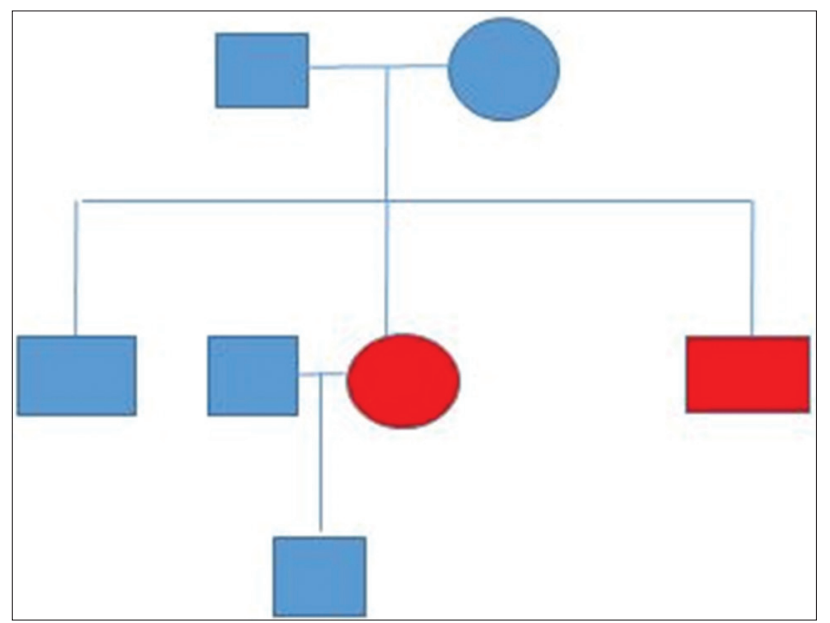

Figure 1: Pedigree chart shows autosomal recessive pattern
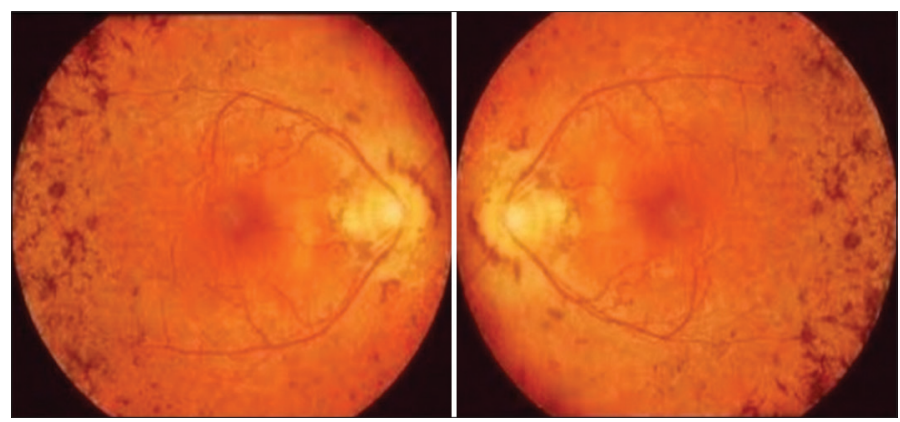

Figure 2: Fundus showed waxy pallor, obliterated cup, arteriolar attenuation, and bony spicules in periphery in both eyes electrically illuminated night-time environment, people can be unaware of a severe loss of rod function because night-time activities are typically done with sufficient light to allow vision with cones. By the time, an individual recognizes the symptom of night blindness, a reduction in cone sensitivity can have happened on top of a loss of rod function. Therefore, a clinician must be cautious when relying on symptoms to identify patients with early RP.

The average age for the diagnosis of RP was reported as 35.1 years by Tsujikawa et al. [6]. Dhanashree et al. reported a similar case of a 32-year-old woman with a chief complaint of difficulty in night vision in her right eye. On examination, the fundus findings were similar to our case and thus, a diagnosis of typical RP was made in the right eye [7].

The classical triad of retinal pigmentary changes comprises the bony spicule, arteriolar attenuation, and waxy disc pallor help in the diagnosis of typical RP [8]. Among the histopathological changes, the earliest histological sign is the shortening of rod outer segments. After photoreceptor cell death, the retinal pigment epithelium becomes detached from the Bruch's membrane and migrates into the neurosensory retina. Accumulation of the retinal pigment epithelial cells in a cuff around retinal vessels leads to bone-spicule pigmentation. In the inner retinal pathology, the waxy disk pallor is due to a thick pre-retinal membrane centered on the disc. Finally, vascular changes of the arteriolar attenuation occur due to increased intravascular oxygen tension [6,9].

At the genetic level, mutations occur due to rhodopsin, peripherin, or abnormal pre-mRNA splicing. Rhodopsin is the protein responsible for initiating the cascade of phototransduction and peripherin protein is involved in the structural integrity of the photoreceptor [10-12].

It is critical to rule out the inflammatory, traumatic, toxic, and cancer-associated retinopathy that can present with retinal pigmentary changes. A number of conditions such as syphilis, rubella, or toxic retinopathies, particularly those caused by due to the intake of drugs such as antimalarial - chloroquine, hydroxychloroquine, antipsychotic - thioridazine, and

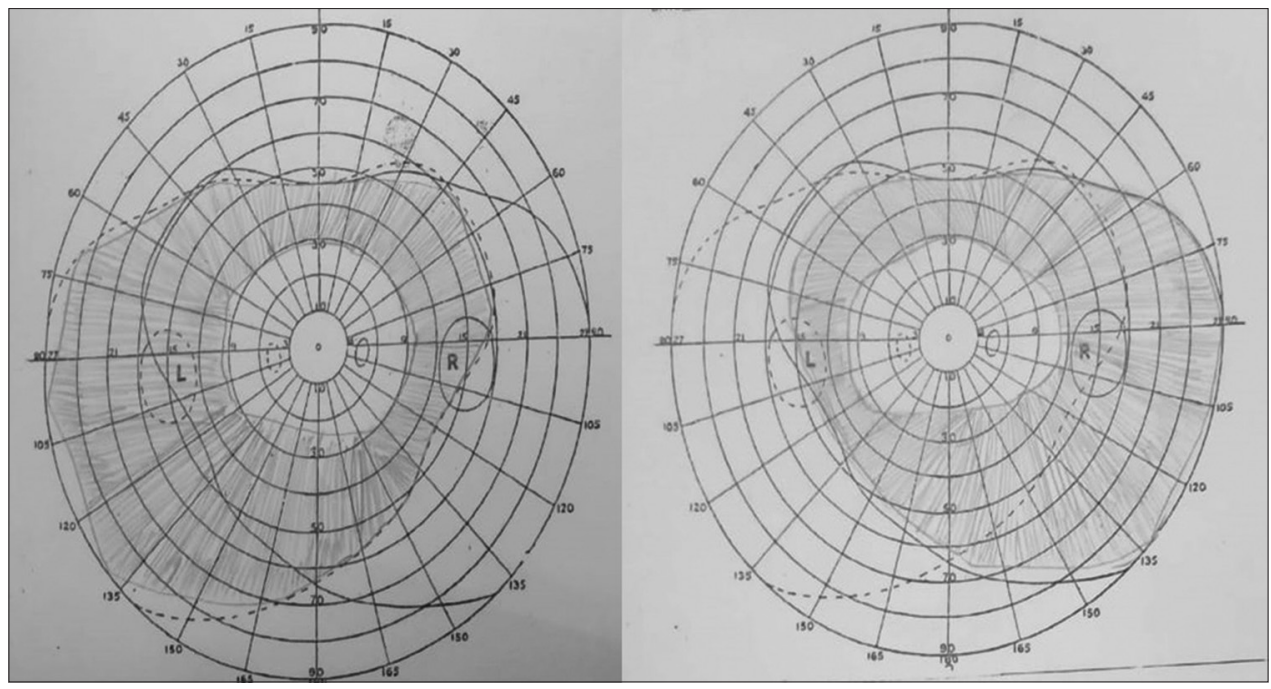

Figure 3: Visual fields on Lister perimetry were suggestive of tubular vision in both eyes 
chlorpromazine, can have a clinical picture that mimics RP which makes it difficult to distinguish from the advanced RP [13]. Furthermore, the time course for vision loss occurs over weeks or months for cancer-associated retinopathy as compared to years with RP [14]. Our patient reported no history consistent with malaria and psychosis or the use of known retinotoxic medications.

\section{CONCLUSION}

Early detection of RP disease is very important which can improve the quality of life of such patients. Furthermore, awareness regarding genetic screening should be raised to decrease the chance of developing RP in future pregnancies.

\section{REFERENCES}

1. Sen P, Bhargava A, George R, Ve Ramesh S, Hemamalini A, Prema R, et al. Prevalence of retinitis pigmentosa in South Indian population aged above 40 years. Ophthalmic Epidemiol 2008;15:279-81.

2. Audo I, Lancelot ME, Mohand-Saïd S, Antonio A, Germain A, Sahel JA, et al. Novel C2orf71 mutations account for approximately $\sim 1 \%$ of cases in a large French arRP cohort. Hum Mutat 2011;32:E2091-103.

3. Lafont E, Manes G, Sénéchal A, Bocquet B, Coustès-Chazalette D, Baudoin $\mathrm{C}$, et al. Patients with retinitis pigmentosa due to RP1 mutations show greater severity in recessive than in dominant cases. J Clin Exp Ophthalmol 2011;2:12.

4. El Shamieh S, Boulanger-Scemama E, Lancelot ME, Antonio A, Démontant V, Condroyer $\mathrm{C}$, et al. Targeted next generation sequencing identifies novel mutations in RP1 as a relatively common cause of autosomal recessive rod-cone dystrophy. Biomed Res Int 2015;2015:485624.
5. Wolfrum U, Nagel-Wolfrum K. Das usher-syndrom, eine ziliopathie des menschen [The usher syndrome, a human ciliopathy]. Klin Monbl Augenheilkd 2018;235:273-80.

6. Tsujikawa M, Wada Y, Sukegawa M, Sawa M, Gomi F, Nishida K, et al. Age at onset curves of retinitis pigmentosa. Arch Ophthalmol 2008;126:337-40.

7. Dhanashree R, Dhileesh P, Aruldas P, Vineet R. Concurrent retinitis pigmentosa and pigmented paravenous retinochoroidal atrophy phenotypes in the same patient. Indian J Ophthalmol 2016;64:775-7.

8. Hamel C. Retinitis pigmentosa. Orphanet J Rare Dis 2006;1:40.

9. Marigo V. Programmed cell death in retinal degeneration: Targeting apoptosis in photoreceptors as potential therapy for retinal degeneration. Cell Cycle 2007;6:652-5.

10. Murray AR, Fliesler SJ, Al-Ubaidi MR. Rhodopsin: The functional significance of asn-linked glycosylation and other post-translational modifications. Ophthalmic Genet 2009;30:109-20.

11. Waseem NH, Vaclavik V, Webster A, Jenkins SA, Bird AC, Bhattacharya SS Mutations in the gene coding for the pre-mRNA splicing factor, PRPF31, in patients with autosomal dominant retinitis pigmentosa. Invest Ophthalmol Vis Sci 2007;48:1330-4.

12. Loewen CJ, Moritz OL, Molday RS. Molecular characterization of peripherin-2 and rom-1 mutants responsible for digenic retinitis pigmentosa. J Biol Chem 2001;276:22388-96.

13. Fornaro P, Calabria G, Corallo G, Picotti GB. Pathogenesis of degenerative retinopathies induced by thioridazine and other antipsychotics: A dopamine hypothesis. documenta ophthalmologica. Adv Ophthalmol 2002;105:41-9.

14. Hoogewoud F, Butori P, Blanche P, Brezin AP. Cancer-associated retinopathy preceding the diagnosis of cancer. BMC Ophthalmol 2018;18:285.

Funding: None; Conflicts of Interest: None Stated.

How to cite this article: Jhajj CS, Kalaivani K, Saranya B, Deepthika P. Detection of asymptomatic retinitis pigmentosa. Indian Journal of Case Report. 2021;7(6):229-231. 